\title{
Individual interviews and focus groups in patients with rheumatoid arthritis: a comparison of two qualitative methods
}

\author{
Michaela Coenen - Tanja A. Stamm • \\ Gerold Stucki • Alarcos Cieza
}

Accepted: 20 May 2011/Published online: 25 June 2011

(C) Springer Science+Business Media B.V. 2011

\begin{abstract}
Purpose To compare two different approaches to performing focus groups and individual interviews, an open approach, and an approach based on the International Classification of Functioning, Disability and Health (ICF). Methods Patients with rheumatoid arthritis attended focus groups $(n=49)$ and individual interviews $(n=21)$. Time, number of concepts, ICF categories identified, and sample size for reaching saturation of data were compared. Descriptive statistics, Chi-square tests, and independent $t$ tests were performed.

Results With an overall time of $183 \mathrm{~h}$, focus groups were more time consuming than individual interviews $(t=9.782$;
\end{abstract}

Michaela Coenen, Gerold Stucki, and Alarcos Cieza-ICF Research Branch in cooperation with the WHO Collaborating Centre for the Family of International Classifications in Germany (at DIMDI). The responsibility for the content of this publication lies with the ICF Research Branch.

M. Coenen · A. Cieza

Institute for Health and Rehabilitation Sciences, Research Unit for Biopsychosocial Health, Ludwig-Maximilians-University, Marchioninistr. 17, 81377 Munich, Germany

T. A. Stamm

Department of Internal Medicine III, Division of Rheumatology, Medical University of Vienna, Währinger Gürtel 18-20, 1090 Vienna, Austria

G. Stucki $(\square)$

Department Health Sciences and Health Policy, University of Lucerne, Pilatusstraße 20, 6000 Luzern 7, Switzerland e-mail: gerold.stucki@paranet.ch

G. Stucki · A. Cieza

Swiss Paraplegic Research (SPF), 6207 Nottwil, Switzerland
$P<0.001)$. In the open approach, 188 categories in the focus groups and 102 categories in the interviews were identified compared to the 231 and 110 respective categories identified in the ICF-based approach. Saturation of data was reached after performing five focus groups and nine individual interviews in the open approach and five focus groups and 12 individual interviews in the ICF-based approach. Conclusion The method chosen should depend on the objective of the study, issues related to the health condition, and the study's participants. We recommend performing focus groups if the objective of the study is to comprehensively explore the patient perspective.

Keywords Focus groups - Individual interviews · Qualitative research - Rheumatoid arthritis - International Classification of Functioning, Disability and Health (ICF)

\section{Introduction}

Qualitative methodology has gained recognition and acceptance in health-science research in recent times [1-3] and is now widely used, particularly in rehabilitation research [4]. Qualitative methods are often included in mixed-methodology studies to obtain a rich and comprehensive view of a research topic [5-7]. Two of the most broadly used techniques in qualitative research are focus groups [8-10] and individual interviews [11, 12]. Unlike quantitative methods, qualitative methods allow individuals to respond in their own words to express their personal categorizations and perceived associations. They are not completely unstructured, since the questions asked have been designed by the researcher to achieve the specific research objectives, and these questions guide the data collection [13]. 
Fig. 1 The biopsychosocial perspective of functioning, disability, and health and the ICF classification. The biopsychosocial model states that functioning and disability are the outcomes of the interaction of health condition and contextual factors

(Environmental Factors and Personal Factors) which take the form of biological, person level, and social level phenomena, grouped by the components Body Functions, Body Structures, and Activities and Participation

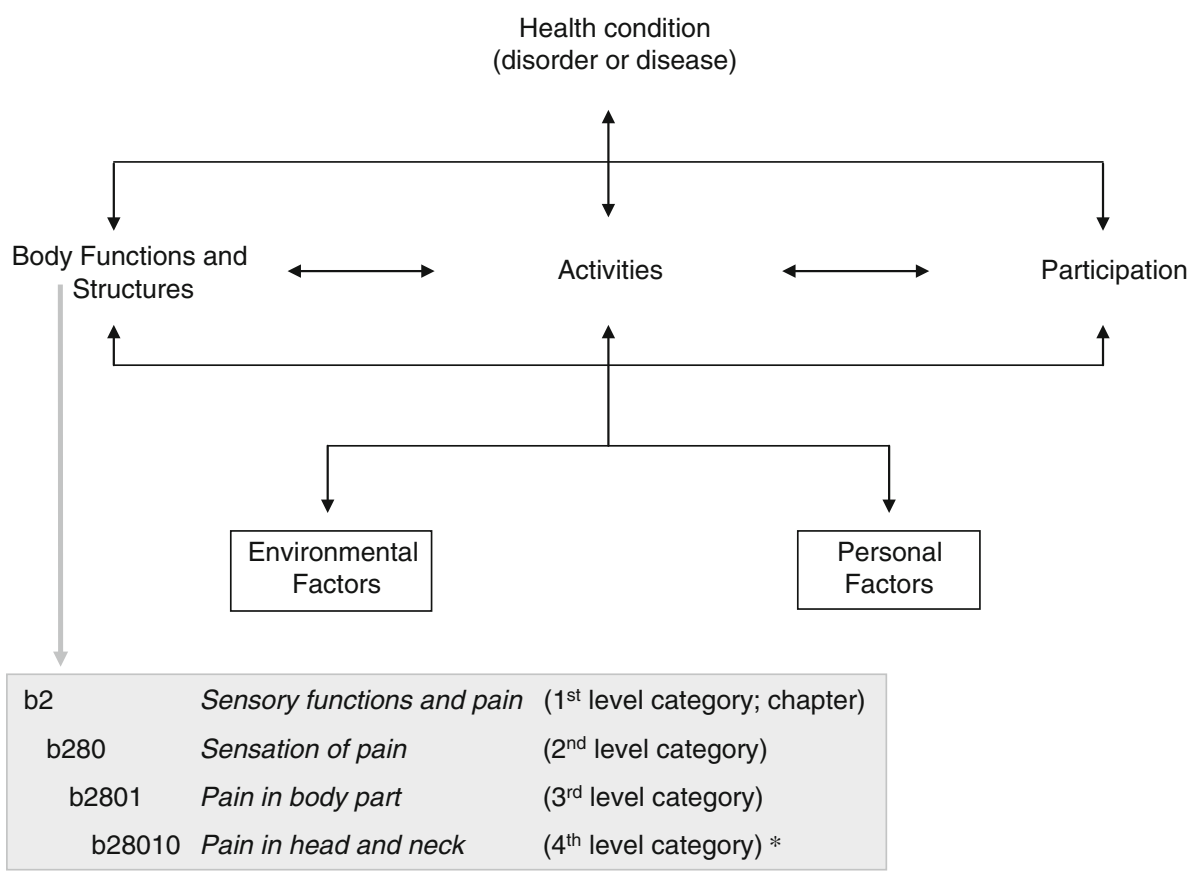

Health condition

disorder or disease)

* only in the components Body Functions and Body Structures
Focus groups generate a rich understanding of people's experiences and beliefs [14]. Carey emphasizes the advantages of the focus-group technique by pointing out that they are "especially well suited for problems in health research where complex clinical issues are often explored through a qualitative approach" (p. 227) [15]. Focus groups differ from individual interviews in that the group interaction enriches the information generated [10, 13]. The idea behind the focus-group methodology is that group processes can help people explore and clarify their views in ways that would be less easily accessible in a one-to-one interview [16]. It is commonly believed that focus groups reveal more ideas and that more information is collected than in individual interviews [17-19]. Focus groups, however, take more time and effort, are more costly in resources, and cause more logistic problems [19].

Another challenge is to compare the results from focus groups and face-to-face interviews, which can be achieved by using a common reference. Specifically, when the information gathered refers to functioning and disability, the International Classification of Functioning, Disability and Health (ICF) [20] (see Fig. 1) can be successfully used to compare results $[21,22]$. The ICF enables a comprehensive description of what patients suffering from certain health conditions experience. The ICF's underlying biopsychosocial model analyses the notions of functioning and disability in terms of the basic components of Body Functions, Body Structures, and Activities and Participation that are outcomes of the interaction between a health condition and Environmental and Personal Factors. The
ICF is constructed according to an etiologically neutral framework, which means that the functioning levels experienced by the individual are not derived directly from the health condition itself, but are described independently. The ICF also provides an internationally recognized language and classification structure useful for comparatively describing functioning and disability at both the individual and the population levels. The ICF contains 1,424 categories organized into four components (the component Personal Factors has not yet been classified). Each ICF category is denoted by a code composed of a letter identifying the components of the classification (b: Body Functions; s: Body Structures; d: Activities and Participation; and e: Environmental Factors) followed by a numeric code starting with the chapter number (one digit), followed by the second level (two digits), and, finally, the third and fourth levels (one digit each). By using the ICF classification as a reference, the complex structure and comprehensive understanding of patients' problems and experiences with a disease or other health condition can be systematically described and explored.

In this study, both qualitative methods, namely focus groups and individual interviews, were used and their efficiency evaluated based on the cost in resources required to perform them. The objective is to compare the efficiency of both focus groups and individual interviews, as well as two different approaches that can be used in both methods, namely an "open approach" and an "ICF-based approach," with regard to (1) formal aspects (costs of the methods in terms of resources) and (2) the results obtained (content). 


\section{Methods}

The study was conducted by means of an audit trail, which is a running log related to a research project. Based on the original study protocol, the audit trail was established by the researchers involved in this study (MC, TS) to document the progress of the study, as well as decisions on methodological topics under discussion. The credibility of the recruitment, data collection, and data analysis of focus groups and individual interviews should be strengthened by applying this audit trail, which also ensured the comparability of the methods and approaches under investigation.

Detailed descriptions of the methods of focus groups and individual interviews have been published elsewhere $[21,22]$.

\section{Design}

We conducted a study with patients suffering from rheumatoid arthritis (RA) comparing two qualitative methods, namely focus groups and individual interviews. The focus groups were performed in Munich and the individual interviews in Vienna. For each of the two methods, two different approaches were used-the so called "open approach" and the "ICF-based approach." In the open approach, open-ended questions were used, such as "If you think about your daily life, what are your problems?", in which the patients were invited to name their problems in terms of the dimensions Body Functions, Body Structures, and Activities and Participation. The patients were also asked about Environmental Factors (barriers and facilitators) influencing their everyday lives (see Table 1). In the ICF-based approach, the titles of the ICF chapters (i.e., first level of the ICF classification) were added to the openended questions (e.g., "Self care: If you think about your daily life, what are your problems in this domain?"). As each chapter was introduced, patients were encouraged to describe any problems they personally experienced related to each specific ICF chapter in their own words. Finally, the patients were asked whether they thought anything was missing in the previous discussion or interview.

\section{Participants}

In focus groups and individual interviews, patients diagnosed with RA according to the revised American College of Rheumatology (ACR) criteria [23] who were willing to participate gave written informed consent according to the Declaration of Helsinki 1996. The study was approved by the institutional review boards of the Medical University of Vienna and the Ludwig-Maximilian University in Munich.

The purposeful sampling of participants followed the maximum-variation strategy [24] based on two criteria: disease duration and age group. These criteria ensure that patients with a broad range in disease duration and age were included in both methods and approaches to provide a comprehensive and diverse description of everyday functioning in RA.

The sample size was determined by saturation of data. Saturation refers to the point at which an investigator has obtained sufficient information from the field [24, 25] (see in detail Data analysis: "Saturation of data").

\section{Data collection}

Focus groups and individual interviews were performed in a non-directive manner according to a standardized guideline including open-ended questions (see Table 1) and further instructions on how to prepare and perform the focus-group and individual-interview sessions, respectively.

At the beginning of each focus group and individual interview, the session's procedure was explained to the participants. Then one of the two different approaches was performed (open approach or ICF-based approach). In the ICF-based approach, the model of the ICF was presented to the participants in layman terms and the titles of the ICF chapters were visualized to highlight the respective ICF chapters. At the end of each focus group and interview, a summary of the main results was given to the participants allowing them to verify and amend emerging issues. The focus groups were conducted by $\mathrm{MC}$ and one group assistant who observed the group process and recorded the data. The individual interviews were conducted by TS. The

Table 1 Open-ended questions in focus groups and individual interviews

\begin{tabular}{ll}
\hline ICF components & Open-ended questions \\
\hline Body Functions & If you think about your body and mind, what does not work the way it is supposed to? \\
Body Structures & If you think about your body, in which parts are your problems? \\
Activities \& Participation & If you think about your daily life, what are your problems? \\
Environmental Factors & If you think about your environment and your living conditions, \\
& - what do you find helpful or supportive? \\
& - what barriers do you experience? \\
\hline
\end{tabular}


focus-group sessions and individual interviews were digitally recorded and transcribed verbatim.

The following variables were recorded for each method and each approach applied:

1. Participants' characteristics

2. Time needed to conduct the studies, especially for the (a) recruitment of patients, (b) preparation of sessions, (c) duration of sessions, (d) transcription, (e) data check of transcripts, (f) data analysis, (g) peer review, and (h) overall time needed to perform the studies

3. Frequencies of concepts identified in the participants' statements

4. Frequencies of ICF categories linked to the identified concepts

5. Number of focus groups and individual interviews needed to reach saturation of data.

Data analysis

The data analysis included the following parts:

\section{Descriptive statistics}

Descriptive statistics of the variables gender, age, disease duration, and time needed to conduct the studies were calculated. Chi-square tests (gender) and independent samples $t$ tests (age, disease duration, time needed to conduct the studies) were performed to explore the differences in participants' characteristics, as well as time required for the two methods and approaches applied. The statistical analyses were performed with SPSS for windows 14.0 (SPSS Inc., Chicago, Illinois, USA).

\section{Qualitative analysis}

Concepts contained in the verbatim transcripts were identified in a multi-level analysis procedure using the "meaning condensation procedure" [11]. In the first step, the transcripts of focus groups and individual interviews were read through to obtain an overview of the collected data. In the second step, data were divided into units of meaning. The theme that dominated a meaning unit was determined. A meaning unit was defined as a specific unit of text containing either a few words or a few sentences with a common theme. Therefore, a meaning-unit division did not follow grammatical rules. The text was divided where the researcher discerned a shift in meaning. In the third step, concepts contained in the meaning units were identified. The frequencies of identified concepts were calculated separately for both methods and approaches applied.

\section{Linking to the ICF}

Concepts which were identified in the qualitative analysis were linked to ICF categories. The linking procedure was performed according to established linking rules [26, 27], which enables a systematic and standardized linking of concepts to ICF categories. Two health professionals trained in the ICF were advised to link each concept to the ICF category representing this concept most precisely based on these linking rules. One concept could be linked to one or more ICF categories, depending on the number of themes contained in the concept. Consensus between the two health professionals was used to decide which ICF category should be linked to each identified concept. If disagreement prevailed, a third person trained in the linking rules was consulted who led a discussion in which the two health professionals who had linked the concepts stated the pros and cons for their linking decisions. The third person then made an informed decision based on these statements. The frequencies of the final agreed-on ICF categories were calculated for the two methods and approaches applied.

\section{Saturation of data}

The sample sizes of both methods and approaches were compared based on the saturation of data. In this study, saturation was defined as the point during data collection and analysis at which the linking of the concepts of two consecutive focus groups or individual interviews revealed no additional second-level ICF categories of the Comprehensive ICF Core Set for RA with respect to previous focus groups and individual interviews. The Comprehensive ICF Core Set for $R A$ is an application of the ICF representing the typical spectrum of problems in functioning of patients with RA [28]. It was developed in a formal decisionmaking and international consensus process integrating evidence collected from preparatory studies [29]. Saturation of data was checked separately for the two methods and approaches applied.

Accuracy of data collection and analysis

The accuracy of the linking procedure was assessed by peer review. Fifteen percent of the transcribed text was randomly selected, analyzed according to the meaning-condensation procedure and linked to the ICF by two health professionals (MC and TS). This process was performed in addition to the process described in the section "Linking to the ICF." The degree of agreement between the two health professionals regarding the identified and linked concepts in this randomly selected text was calculated by kappa 
statistics with $95 \%$ bootstrapped confidence intervals. The results of the peer review were reported elsewhere [21, 22].

Several strategies and procedures were used to assure the comparability of both methods and approaches under investigation. The study progress was documented with the audit trail. A standardized guideline including open-ended questions and further instructions on data collection and analysis was used to perform the study. A predefined recruitment strategy using maximum variation and criteria for saturation of data was mandatory for both methods and approaches. The linking of the concepts was performed based on established linking rules and checked by peer review.

\section{Results}

\section{Characteristics of participants}

Forty-nine patients (open approach: $n=25$; ICF-based approach: $n=24$ ) and 21 patients (open approach: $n=13$; ICF-based approach: $n=8$ ) participated in the focus groups and individual interviews, respectively. Participants' characteristics are summarized in Table 2.

Time needed to conduct the studies

Table 3 shows the time needed to perform the different aspects of the study in detail (mean, SD, results of independent $t$ test, $P$ values) for the two methods and approaches applied.

Identified concepts: results of the qualitative analysis

In the focus groups, 897 (open approach) and 1,003 concepts (ICF-based approach) were identified. In the individual interviews, 522 concepts were identified in the open approach and 374 in the ICF-based approach.

Identified ICF categories: results of the linking procedure

In the focus groups, a total of 188 ICF categories in the open approach and 231 ICF categories in the ICF-based

Table 2 Characteristics of the participants

\begin{tabular}{|c|c|c|c|c|}
\hline \multirow[t]{2}{*}{ Characteristics } & \multicolumn{2}{|l|}{ Focus groups } & \multicolumn{2}{|c|}{ Individual interviews } \\
\hline & Open approach & ICF-based approach & Open approach & ICF-based approach \\
\hline \multicolumn{5}{|l|}{ Age (years) } \\
\hline $\mathrm{M}(\mathrm{SD})$ & $59.0( \pm 14.9)$ & $54.3( \pm 12.9)$ & $58.7( \pm 13.7)$ & $57.0( \pm 15.6)$ \\
\hline Range & $24-81$ & $35-75$ & $30-79$ & $25-73$ \\
\hline Comparison of methods ${ }^{\mathrm{a}}$ & \multicolumn{4}{|c|}{$t=-0.262 ; P=0.794^{\mathrm{c}}$} \\
\hline Comparison of approaches ${ }^{b}$ & \multicolumn{2}{|c|}{$t=1.172 ; P=0.247$} & \multicolumn{2}{|c|}{$t=0.170 ; P=0.867$} \\
\hline \multicolumn{5}{|l|}{ Gender (female) } \\
\hline$n(\%)$ & $22(88.0)$ & $20(83.3)$ & $11(84.6)$ & $7(87.5)$ \\
\hline Comparison of methods & \multicolumn{4}{|c|}{$\chi^{2}=0.000 ; P=1.000^{\mathrm{d}}$} \\
\hline Comparison of approaches & \multicolumn{2}{|c|}{$\chi^{2}=0.218 ; P=0.641$} & \multicolumn{2}{|c|}{$\chi^{2}=0.034 ; P=0.854$} \\
\hline \multicolumn{5}{|l|}{ Duration of disease (years) } \\
\hline $\mathrm{M}(\mathrm{SD})$ & $15.8( \pm 10.4)$ & $14.6( \pm 12.5)$ & $9.7( \pm 10.6)$ & $11.7( \pm 8.8)$ \\
\hline Range & $4-38$ & $3-36$ & $1-29$ & $1-26$ \\
\hline Comparison of methods & \multicolumn{4}{|c|}{$t=1.711 ; P=0.092$} \\
\hline Comparison of approaches & \multicolumn{2}{|c|}{$t=0.350 ; P=0.728$} & \multicolumn{2}{|c|}{$t=-0.493 ; P=0.628$} \\
\hline \multicolumn{5}{|l|}{ Occupation $[n(\%)]$} \\
\hline Paid work & $5(20.0)$ & $6(25.0)$ & $2(15.4)$ & - \\
\hline Unpaid work & - & $1(4.2)$ & - & $1(12.5)$ \\
\hline Unemployed & $2(8.0)$ & $2(8.3)$ & $1(7.7)$ & $1(12.5)$ \\
\hline Retired & $16(64.0)$ & $14(58.3)$ & $9(69.2)$ & $6(75.0)$ \\
\hline Homemaker & $2(8.0)$ & $1(4.2)$ & $1(7.7)$ & - \\
\hline
\end{tabular}

${ }^{a}$ Comparison of methods (focus groups vs. individual interviews) irrespective of the approach

${ }^{\mathrm{b}}$ Comparison of approaches (open vs. ICF-based approach); calculated for focus groups and individual interviews, respectively

${ }^{\mathrm{c}}$ Independent samples $t$ test for comparison of methods $(d f=29)$ and comparison of approaches $(d f=8$ for focus groups; $d f=19$ for individual interviews), respectively

${ }^{\mathrm{d}}$ Chi-square test according to Pearson $(d f=1)$ 


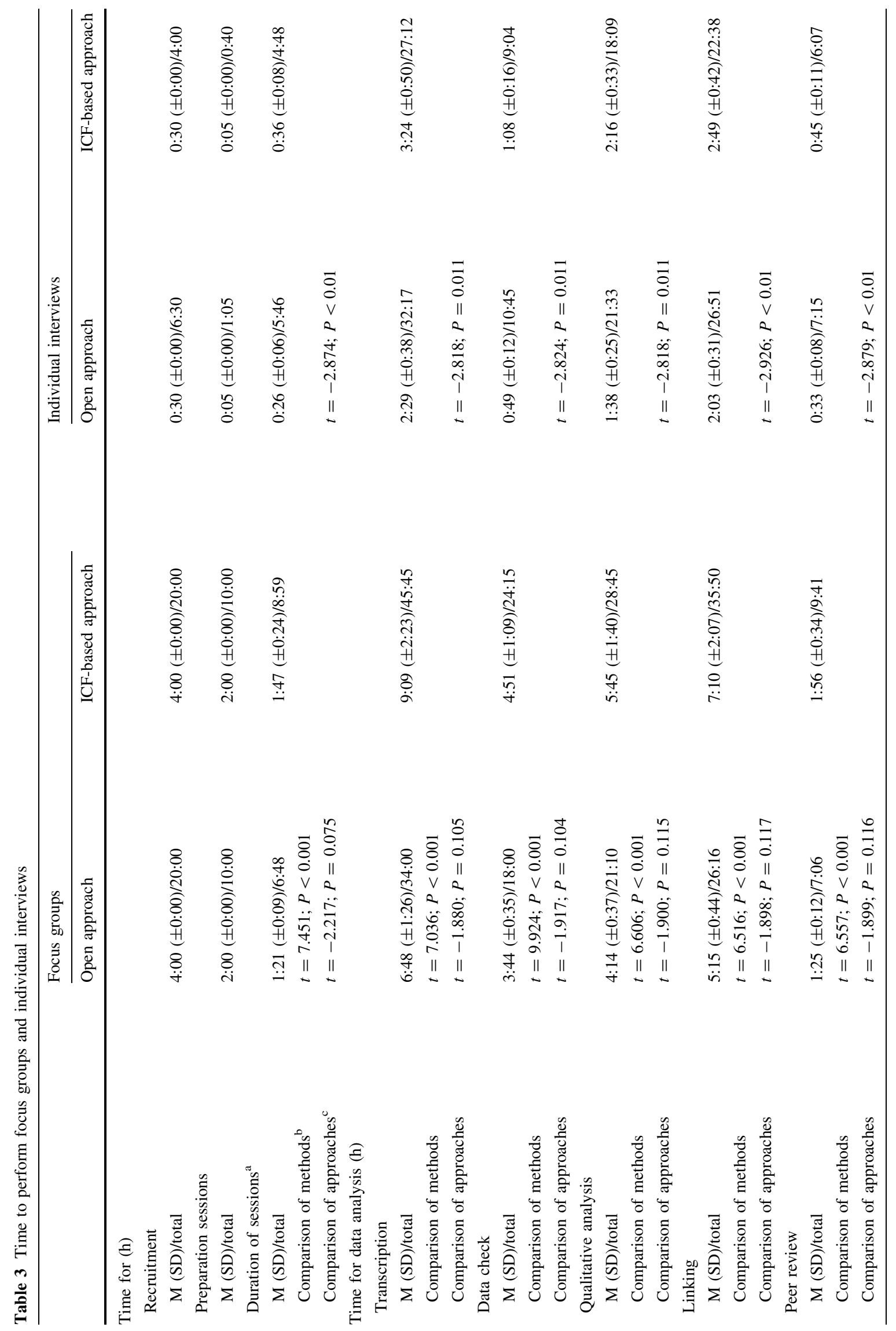




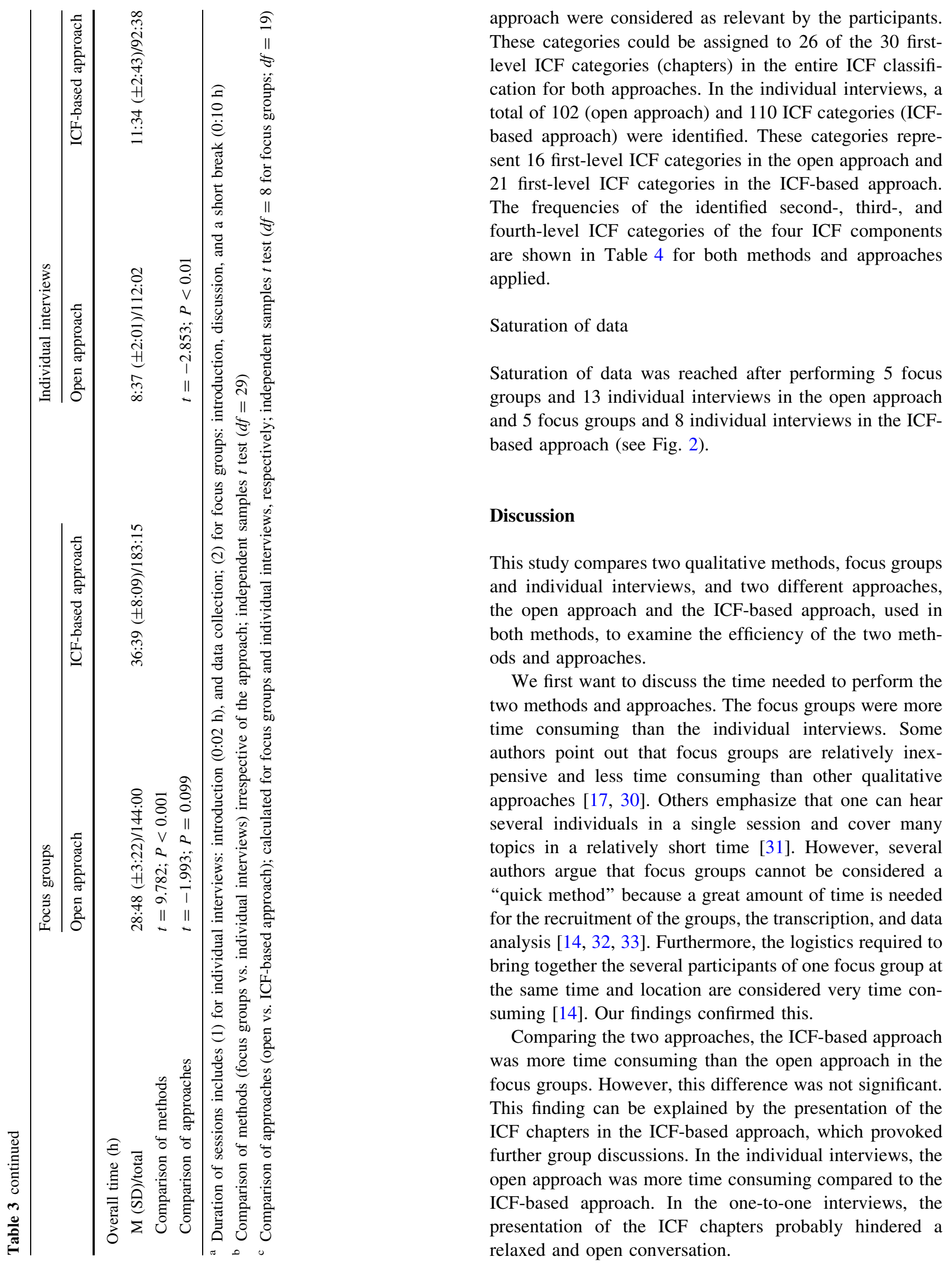


Table 4 Identified ICF categories in focus groups and individual interviews
${ }^{\text {a }}$ Total number of ICF categories without the "other specified" ICF categories (uniquely identified by the final code number 8 ) and the "unspecified" ICF categories (uniquely identified by the final code number 9). The ICF categories "other specified" and "unspecified" were excluded in the reported summation

\begin{tabular}{|c|c|c|c|c|}
\hline \multirow[t]{2}{*}{ ICF categories } & \multicolumn{2}{|l|}{ Focus groups } & \multicolumn{2}{|c|}{ Individual interviews } \\
\hline & Open approach & $\begin{array}{l}\text { ICF-based } \\
\text { approach }\end{array}$ & Open approach & $\begin{array}{l}\text { ICF-based } \\
\text { approach }\end{array}$ \\
\hline \multicolumn{5}{|l|}{ Body Functions } \\
\hline 2nd-level ICF categories ${ }^{a}$ & 26 & 39 & 6 & 9 \\
\hline 3rd-level ICF categories & 14 & 32 & 11 & 14 \\
\hline 4th-level ICF categories & 5 & 9 & 2 & 2 \\
\hline \multicolumn{5}{|l|}{ Body Structures } \\
\hline 2nd-level ICF categories & 8 & 9 & 2 & 5 \\
\hline 3rd-level ICF categories & 11 & 12 & 5 & 8 \\
\hline 4th-level ICF categories & 9 & 10 & 5 & 2 \\
\hline \multicolumn{5}{|l|}{ Activities \& Participation } \\
\hline 2nd-level ICF categories & 18 & 24 & 18 & 14 \\
\hline 3rd-level ICF categories & 46 & 48 & 26 & 29 \\
\hline \multicolumn{5}{|l|}{ Environmental Factors } \\
\hline 2nd-level ICF categories & 30 & 28 & 15 & 15 \\
\hline 3rd-level ICF categories & 21 & 20 & 12 & 12 \\
\hline \multicolumn{5}{|l|}{ Total } \\
\hline 2nd-level ICF categories & 82 & 100 & 41 & 43 \\
\hline 3rd-level ICF categories & 92 & 112 & 54 & 63 \\
\hline 4th-level ICF categories & 14 & 19 & 7 & 4 \\
\hline
\end{tabular}



Fig. 2 Saturation of data in focus groups and individual interviews

Besides the time-related aspects of this study, the content-related issues of the study - the results of the qualitative analysis and the identified concepts-are also pertinent. In the qualitative analysis, more concepts were identified in focus groups compared to individual interviews. It could be assumed that the interactive nature of the focus-group sessions helped the participants to comprehensively explore their views on functioning in everyday life. The following example highlights this.

Participant A: "I used to go to sports very often. Now I can't anymore. I even had to quit swimming."

Participant B: "Exactly! I also had to quit swimming."

Participant C: "Swimming? For me the problem is: I can no longer cycle."
Comparing the two approaches, more concepts were identified in the ICF-based focus-group approach. The larger number of concepts identified in the ICF-based approach could be associated with the naming of the ICF chapters in the open-ended questions. Because of the wordings of these questions, the participants could be encouraged to name experiences and problems in functioning which they may not have mentioned spontaneously in the open approach.

It is also interesting to compare these results to those of Rat et al. [34], who performed a study on item-generation for quality-of-life instruments using individual interviews and focus groups. The authors stated that the different methods applied-(1) semi-structured interviews, including spontaneous conversation and probing to identify further issues, (2) cognitive interviews, and (3) unstructured focus groups-were not equivalent in generating qualityof-life-related items. In contrast to the results of our study, persons who participated in the semi-structured interviews and cognitive interviews produced a larger number of items than persons participating in the focus groups. These differences in the results can be explained by the fact that Rat and colleagues performed two focus groups without reaching saturation of data based on a very open approach without providing hints to the participants.

Coming back to the results of our study, it is important to mention that some patient-sensitive issues were only reported in the ICF-based approach (e.g., sexual functions, toileting). However, this result was not confirmed by the 
individual interviews, where more concepts were also retrieved from the open approach. One could reason that, in individual interviews, the participants felt free to talk when responding to open-ended questions without being provided with additional hints. They jumped at the chance to report on many issues related to their everyday functioning.

Regarding the results of the linking procedure, this article demonstrates that the ICF classification is useful to compare the results of focus groups and individual interviews when the topic of interest is functioning and disability. More ICF categories were identified in focus groups than in individual interviews. In addition, more ICF categories were revealed by the ICF-based approach in both methods (focus groups and individual interviews). We found more ICF categories in several levels of the classification (second to fourth levels) in the focus groups than in the individual interviews. Thus, the precision of statements and ICF categories was higher in the focus-group data than in the data derived from individual interviews.

This result supports the popular assumption that group processes can help people to explore and clarify their views [16]. The non-directive nature of focus groups allows participants to comment, explain, disagree, and share experiences and attitudes [35]. The literature points out that there are both greater synergy and spontaneity, which optimizes the generation of (new) ideas, in focus groups $[10,13,19]$. Participants' statements in focus groups could be seen as stimuli for other participants to voice similar experiences or problems in the discussion. Therefore, a more relaxed atmosphere can be established in focus groups than in a one-to-one setting. Focus-group participants do not feel forced to answer every question [9]. Feeling comfortable and free to speak in a "safe" forum facilitates self-disclosure [14, 36]. This more relaxed atmosphere in focus groups could create a setting where sensitive topics can be discussed more frequently and openly than in other qualitative methods [31, 37].

In contrast, Greenbaum emphasizes that sensitive topics should be addressed more often in individual interviews avoiding the possible embarrassment of individuals in a focus-group setting [38]. However, the participants of our focus groups, as well as the participants of the individual interviews, named several sensitive topics (e.g., weight maintenance, urination functions, and intimate relationships). Some authors argue that potential differences in the amount of information gathered in focus groups and individual interviews and the willingness of individuals to discuss personal topics depend on the research topic and group composition [38, 39]. Ezzy [40] concludes that the type of information revealed in focus groups is different from that obtained in individual interviews. The participants' statements revealed in focus groups and the interpretation of these statements are profoundly influenced by the group processes and the relationships among the participants, whereas statements in individual interviews are influenced by the relationship between the interviewer and the interviewee. We cannot confirm any difference in the type of information gained between the two qualitative methods applied.

The number of sessions required to reach saturation of data must also be taken into account when examining the efficiency of the methods applied. Saturation of data was reached earlier in the focus groups than in the individual interviews. According to the conventions of qualitative data analysis, the unit of data analysis and the unit for applying saturation of data are the focus group and not the individual group participant [41, 42]. In our study, more patients participated in the focus groups than in the individual interviews. Our results are comparable to those of Guest et al., who report that $73 \%$ and $92 \%$ of all identified codes in a total of 30 interviews are found after conducting the first 6 and first 12 individual interviews, respectively.

To ensure that the differences found in the results discussed above are not related to participant characteristics, we compared these among the methods and approaches applied and found no differences. The participants' characteristics (gender, age, disease duration) were comparable to those in other German, Austrian [43, 44], and international samples [45, 46].

One has to consider potential "hidden costs" associated with the planning and performing of focus groups, which were not systematically assessed in this study and were not reported in the "Results" section. However, these hidden costs might substantially increase the amount of time needed to plan and perform a study. Finding an appropriate and convenient location can be more difficult in focus groups compared to individual interviews [14]. A focus group's moderator has to fulfill more qualifications compared to the interviewee because the one-to-one setting is generally considered to be easier to handle than a group session [33, 37].

The recruitment strategy of participants (e.g., availability) has to be carefully planned to ensure an unproblematic sampling. Over-recruiting has been reported as beneficial [47], as some potential participants could miss the fixed date of the focus-group session. The task of transcribing the recordings of the focus groups should be done by a highly experienced person because it is especially labor intensive and often challenging [48]. Distinguishing between participants talking at the same time, softly spoken statements, and unintelligible pronunciations can make transcription difficult.

In our study, the hidden costs of the focus groups were comparatively low. We used the facilities and equipment of the medical department without time-consuming logistic problems. Pre-existing lists of potential participants with 
documentation of addresses, telephone numbers, and age were available in the focus-group study. The participants in the focus groups were easily found in the rheumatology day clinic. Thus, the effort expended for support and contact (e.g., additional telephone calls, letters, and information) was relatively small. Participants' willingness and trustworthiness were remarkably high. Therefore, we did not need to over-recruit participants for the focus groups. We could fall back on a highly experienced person for the transcriptions of the recordings, who performed this task extraordinarily quickly and precisely.

Although quantitative analyses are usually not used in qualitative studies, these qualitative data can be used for future explorative statistical analyses applying quantitative methods, such as Rasch analyses. The categories reported in Table 4, for instance, can be scored as present or absent for each focus group and interview and then scaled using a Rasch model [49, 50]. Given that the resulting scores provide sufficient statistics, the individual and by-group measures could be used for comparing the different amounts of information obtained from each method. However, the results of such analyses can only be considered explorative and interpreted with caution due to the small sample size.

\section{Limitations of the study}

There are some limitations in this study that must be discussed. Participants' recruitment for the focus groups and individual interviews was performed at two different locations (Munich and Vienna). To ensure rigor regarding participant selection purposeful sampling following a maximum-variation strategy with defined criteria was applied.

The results of both methods and approaches were compared based on the number of identified concepts and linked ICF categories. More concepts and identified ICF categories do not necessarily indicate greater understanding and comprehensive information given by the participants.

Additionally, the reported time was the working time required to perform and analyze the sessions without adding the time needed, for example, for logistics. One also has to take into account that all health professionals involved in the studies had expert knowledge and experience in the application of the ICF and in performing and analyzing qualitative studies.

Involving participants with other health conditions might have produced conflicting findings in the comparison of the results between focus groups and individual interviews and saturation of data. Therefore, similar studies with different patient groups are needed.

Some detailed participants' statements were summarized on a higher level of abstraction (e.g., "opening a milk package", "using a coffee machine", and "using one's hand while sailing" were linked to the ICF category "hand and arm use"). Data precision could have been underestimated as a consequence of this aggregation. However, the linking procedure enabled comparison of the results of the two methods and approaches applied in this study.

Saturation of data operationalized by the cumulative frequencies of second-level ICF categories might be a questionable criterion to determine the number of focus groups and participants. It might be impossible to obtain sufficient information from the field, especially in individual interviews, because adding one participant after two interviews, which did not reveal any new information, could still add some more issues from the individual's perspective. In addition, we are aware that the higher number of identified ICF categories revealed in the focus groups could also be related to the number of participants included. The inclusion of further participants in the individual interviews could probably increase the number of identified ICF categories, whereas the reported data saturation of the individual interviews contradicts this argument.

Finally, this study compared two methods and approaches, and since this comparison is explorative, the results of these differences must be interpreted with caution.

\section{Conclusion}

For a closing examination of the efficiency of two qualitative methods (focus groups and individual interviews) and two approaches (open approach and ICF-based approach), the time and effort expended and the results obtained must be weighted under consideration of the sample size. In any case, more time is needed to organize a focus-group session. The performance of focus groups, and especially the ICF-based approach, was more time consuming compared to the individual interviews and the open approach, respectively. Focus groups and the ICF-based approach generated more ICF categories. This can be relevant if the patient perspective is to be explored in depth. Thus, the final recommended approach should not only be superior concerning the precision of data generated and the amount of concepts identified (bandwidth), but also concerning the feasibility and economic aspects of the applied procedure. The decision about the favored method should depend on the study objective, issues related to the health condition, and the participants involved in the study. In conclusion, researchers using qualitative methods should balance the costs, advantages, and disadvantages of available methods under consideration of the study objective before beginning the study.

For further research, we recommend performing focus groups if the study objective is to comprehensively explore functioning and health from the patient perspective. 
Individual interviews are the preferred method to obtain a rough overview of aspects of functioning and health.

Acknowledgments The performance of the focus groups was funded by the German self-help organization "Deutsche Rheuma-Liga e.V.-Bundesverband." We thank Mrs. Elke Ruschek and Mrs. Sieglinde Stamm for their competent and precise transcribing of the focus groups and individual interviews. We would like to thank all patients who participated in the study.

\section{References}

1. Giacomini, M., \& Cook, D. (2000). Users' guides to the medical literature: XXIII. Qualitative research in health care, a. Are the results of the study valide. Journal of the American Medical Association, 284, 357-362.

2. Mays, N., \& Pope, C. (2000). Qualitative research in health care: Assessing quality in qualitative research. British Medical Journal, 320, 50-52.

3. Murphy, E., Dingwall, R., Greatbatch, D., Parker, S., \& Watson, P. (1998). Qualitative research methods in health technology assessment: A review of the literature (Vol. 2, no. 16). Southampton: Health Technology Assessment.

4. Oehman, A. (2005). Qualitative methodology for rehabilitation research. Journal of Rehabilitation Medicine, 37, 273-280.

5. Moffatt, S., White, M., Mackintosh, J., \& Howel, D. (2007). Using quantitative and qualitative data in health service research-what happens when mixed method findings conflict? Health Service Research, 6. doi: 10.1186/1472-6963-1186-1128.

6. Fern, E. (1982). The use of focus groups for idea generation: The effects of group size, acquaintanceship, and moderator on response quantity and quality. Journal of Marketing Research, 19(1), 1-13.

7. Kroll, T., Neri, M., \& Miller, K. (2005). Using mixed methods in disability and rehabilitation research. Rehabilitation Nursing, 30(3), 106-113.

8. Morgan, D. (1997). Focus groups as qualitative research (2nd ed.). Newbury Park: Sage Publications.

9. Vaughn, S., Schumm, J., \& Sinagub, J. (1996). Focus group interviews in education and psychology. Thousand Oaks: Sage Publications.

10. Sim, J. (1998). Collecting and analysing qualitative data: Issues raised by the focus group. Journal of Advanced Nursing, 28(2), 345-352.

11. Kvale, S. (1996). Interviews - an introduction to qualitative research interviewing. California: Sage.

12. Wengraf, T. (2001). Qualitative research interviewing: Biographic narratives and semi-structured methods. London: Sage.

13. Stewart, D., \& Shamdasani, P. (1990). Focus groups: Theory and practice. Newbury Park: Sage.

14. Morgan, D. (1998). The focus group guidebook. Focus group kit I. Thousand Oak: Sage.

15. Carey, M. (Ed.). (1994). Issues in qualitative research methods. Thousand Oaks: Sage.

16. Kitzinger, J. (1995). Qualitative research. Introducing focus groups. British Medical Journal, 311(7000), 299-302.

17. Morgan, D. (1988). Focus groups as qualitative research. London: Sage.

18. Kingry, M., Tiedje, L., \& Friedman, L. (1990). Focus groups: A research technique for nursing. Nursing Research, 39(2), 124-125.

19. Thomas, L., MacMillan, J., McColl, E., Hale, C., \& Bond, S. (1995). Comparison of focus group and individual interview methodology in examining patient satisfaction with nursing care. Social Sciences in Health, 1(4), 206-220.
20. World Health Organisation. (2001). International Classification of Functioning, Disability and Health: ICF. Geneva: WHO.

21. Coenen, M., Cieza, A., Stamm, T., Amann, E., Kollerits, B., \& Stucki, G. (2006). Validation of the International Classification of Functioning, Disability and Health (ICF) Core Set for rheumatoid arthritis from the patient perspective using focus groups. Arthritis Research \& Therapy, 8, R84. doi:10.1186/ar1956.

22. Stamm, T., Cieza, A., Coenen, M., Machold, K., Nell, V., Smolen, J., et al. (2005). Validating the International Classification of Functioning, Disability and Health Comprehensive Core Set for rheumatoid arthritis from the patient perspective: A qualitative study. Arthritis and Rheumatism, 53(3), 431-439.

23. Arnett, F., Edworthy, S., Bloch, D., McShane, D., Fries, J., Cooper, N., et al. (1988). The American rheumatism association 1987 revised criteria for the classification of rheumatoid arthritis. Arthritis and Rheumatism, 31(3), 315-324.

24. Patton, M. (1990). Qualitative evaluation and research methods (2nd ed.). Newbury Park: Sage Publications.

25. Krueger, R., \& Casey, M. (2000). Focus groups: A practical guide for applied research. Thousand Oaks: Sage.

26. Cieza, A., Geyh, S., Chatterji, S., Kostanjsek, N., Ustun, B., \& Stucki, G. (2005). ICF linking rules: An update based on lessons learned. Journal of Rehabilitation Medicine, 37(4), 212-218.

27. Cieza, A., Brockow, T., Ewert, T., Amman, E., Kollerits, B., Chatterji, S., et al. (2002). Linking health-status measurements to the International Classification of Functioning, Disability and Health. Journal of Rehabilitation Medicine, 34(5), 205-210.

28. Cieza, A., \& Stucki, G. (2004). New approaches to understanding the impact of musculoskeletal conditions. Best Practice \& Research in Clinical Rheumatology, 18(2), 141-154.

29. Stucki, G., Cieza, A., Geyh, S., Battistella, L., Lloyd, J., Symmons, D., et al. (2004). ICF Core Sets for rheumatoid arthritis. Journal of Rehabilitation Medicine, 44(Suppl), 87-93.

30. Thornton, C. (1996). A focus group inquiry into the perceptions of primary health care teams and the provision of health care for adults with a learning disability living in the community. Journal of Advanced Nursing, 23(6), 1168-1176.

31. Basch, C. (1987). Focus group interviews: An under-utilized research technique for improving theory and practice in health education. Health Education Quarterly, 14(4), 411-448.

32. Krueger, R. (1995). The future of focus groups. Qualitative Health Research, 5, 524-530.

33. Parsons, M., \& Greenwood, J. (2000). A guide to the use of focus groups in health care research: Part 1. Contemporary Nurse, 9(2), 169-180.

34. Rat, A.-C., Pouchot, J., Guillemin, F., Baumann, M., Retel-Rude, N., Spitz, E., et al. (2007). Content of quality-of-life instruments is affected by item-generation methods. International Journal for Quality in Health Care, 19(6), 390-398.

35. Powell, R., Single, H., \& Lloyd, K. (1996). Focus groups in mental health research: Enhancing the validity of user and provider questionnaires. International Journal of Social Psychiatry, 42, 193-206.

36. Coté-Arsenault, D., \& Morrison-Beedy, D. (2005). Maintaining your focus in focus groups: Avoiding common mistakes. Research in Nursing and Health, 28, 172-179.

37. Carey, A. (1994). The group effect in focus groups: Planning, implementing, and interpreting focus group research. In J. Morse (Ed.), Critical issues in qualitative research methods (pp. 225-241). Thousand Oaks: Sage.

38. Greenbaum, T. (2000). Moderating focus groups. Thousand Oaks: Sage Publications.

39. Kitzinger, J., \& Barbour, R. (Eds.). (1999). Introduction: The challenge and promise of focus groups (Vol. 1-20). London: Sage. 
40. Ezzy, D. (2001). Are qualitative methods misunderstood? Australian and New Zealand Journal of Public Health, 25(4), 294-297.

41. Ward, V., Bertrand, J., \& Brown, L. (1994). The comparability of focus groups and survey results. Evaluative Reviews, 15(2), 266-283.

42. Clarke, A. (1999). Focus group interviews in health-care research. Professional Nurse, 14(6), 395-397.

43. Westhoff, G., Listing, J., \& Zink, A. (2000). Loss of physical independence in rheumatoid arthritis: Interview data from a representative sample of patients in rheumatologic care. Arthritis Care \& Research, 13, 11-22.

44. Zink, A., Listing, J., Klindworth, C., \& Zeidler, H. (2001). The national database of the german collaborative arthritis centres: I. Structures, aims, and patients. Annals of the Rheumatic Diseases, 60, 199-206.

45. Ahlmén, M., Nordenskiöld, U., Archenholtz, B., Thyberg, I., Rönnqvist, T., Lindén, L., et al. (2005). Rheumatology outcomes:
The patient's perspective. A multicentre focus group interview study of Swedish rheumatoid arthritis patients. Rheumatology, 44, $105-110$.

46. Albers, J., Kuper, H., van Riel, P., Prevoo, M., 't Hof, M., van Gestel, A., et al. (1999). Socio-economic consequences of rheumatoid arthritis in the first years of the disease. Rheumatology, $38,423-430$.

47. Jackson, P. (1998). Focus group interviews as a methodology. Nurse Researcher, 6(1), 72-84.

48. Clark, J. M., Maben, J., \& Jones, K. (1996). The use of focus group interviews in nursing research: Issues and challenges. Nursing Times Research, 1(2), 143-153.

49. Andrich, D. (1988). Rasch models for measurement. Newbury Park, CA: Sage.

50. Rasch, G. (1992). Probabilistic models for some intelligence and attainment tests. Chicago: MESA Press. 